\title{
Computation Intelligence in Medicine-Data Analysis and Modelling
}

\author{
Ewa Pietka \\ Silesian University of Technology, Institute of Electronics, 44-100 Gliwice, Poland \\ Correspondence should be addressed to Ewa Pietka, epietka@polsl.pl \\ Received 31 December 2008; Accepted 31 December 2008 \\ Copyright (C) 2008 Ewa Pietka. This is an open access article distributed under the Creative Commons Attribution License, which \\ permits unrestricted use, distribution, and reproduction in any medium, provided the original work is properly cited.
}

In clinical application, we deal with problems which have to be solved in a fast and objective way. However, human observation is influenced by internal (coming form the observer) as well as external (often independent from the observer) impacts. The objectivity of classification is restricted by the receptivity of human senses which are influenced by the experiences or level of training, psychological conditions (tiredness, hurry, etc.), as well as external conditions (lighting, destructive noise, etc.). The failure in perception questions the entire recognition process. The recognition process itself, influenced also by the abovementioned conditions, may cause a slow down and/or lead to a false diagnosis.

New computerized approaches to various problems have become critically important in healthcare. Mathematical information analysis, modelling, and computer simulation become standard tools underpinning the current rapid progress with developing computational intelligence. We are witnessing a radical change as technologies have been integrated into systems that address the core of medicine, including patient care in ambulatory and in-patient setting, disease prevention, health promotion, rehabilitation, and home care. Computational intelligence is in widespread use for the support of patient medical diagnosis and treatment, the assessment of the quality of care, and the enhancement of decision making, modelling, simulation, and medical research. A computerized support in the analysis of patient information and implementation of a computer-aided diagnosis and treatment systems increases the objectivity of the analysis and speeds up the response to pathological changes.

This special issue consists of 5 articles. The subsequent papers are organized into 3 groups.

The first one employs mathematical tools in the data analysis. A computer-aided diagnosis system for breast cancer has been presented by Abdel-Qader and Abu-Amara. They have implemented the independent component analysis and fuzzy classifier to identify and label suspicious regions in mammograms.

An estimation methodology is presented by Mital and Pidaparti to determine the breast tumor parameters using the surface temperature profile that may be obtained by infrared thermography. The estimation methodology involves evolutionary algorithms using artificial neural network and genetic algorithm. The artificial neural network is used to map the relationship of tumor depth, tumor size, and the heat generation to the temperature profile over the idealized breast model. The genetic algorithm estimates the tumor parameters (depth, size, and heat generation) by minimizing a fitness function involving the temperature profiles obtained from simulated data or clinical data.

The second group has employed a modelling technique as a support in the assessment or decision-making problem.

Rau et al. have implement the computational fluid dynamics techniques to investigate the hemodynamic effect of unequal anterior cerebral artery flow rates on the anterior cerebral and anterior communicating artery (ACA-ACOM) bifurcations. Using an idealized 2D symmetric model of the ACA-ACOM geometry, the flow field and wall shear stress (WSS) at the bifurcation regions are assessed for pulsatile inflows with left to right flow ratios.

A model-based approach to reproduce individual heart rate signals acquired during tilt tests is proposed by Le Rolle et al. A new physiological model adapted to this problem and coupling the autonomic nervous system, the cardiovascular system, and global ventricular mechanics is presented. Evolutionary algorithms are used for the identification of patient-specific parameters, in order to reproduce heart rate 
signals obtained during tilt tests. The proposed approach is able to reproduce the main components of the observed heart rate signals and represents a first step toward a model-based interpretation of these signals.

The third group, employed in orthopedics, develops experimental and numerical methods to explore the stresses generated around the implants and bone screws. Chaudhary et al. have presented a finite-element model of a human mandible created with a fixated fracture in the parasymphyseal region. The mandibular model has then been anatomically loaded. Next, the forces exerted by the fixation plate onto the simplified screws are obtained and transferred to another finite-element submodel of a screw implant embedded in a trilaminate block with material properties of cortical and cancellous bone. The stress in the bone surrounding the screw implant has been compared for different screw configurations.

Ewa Pietka 

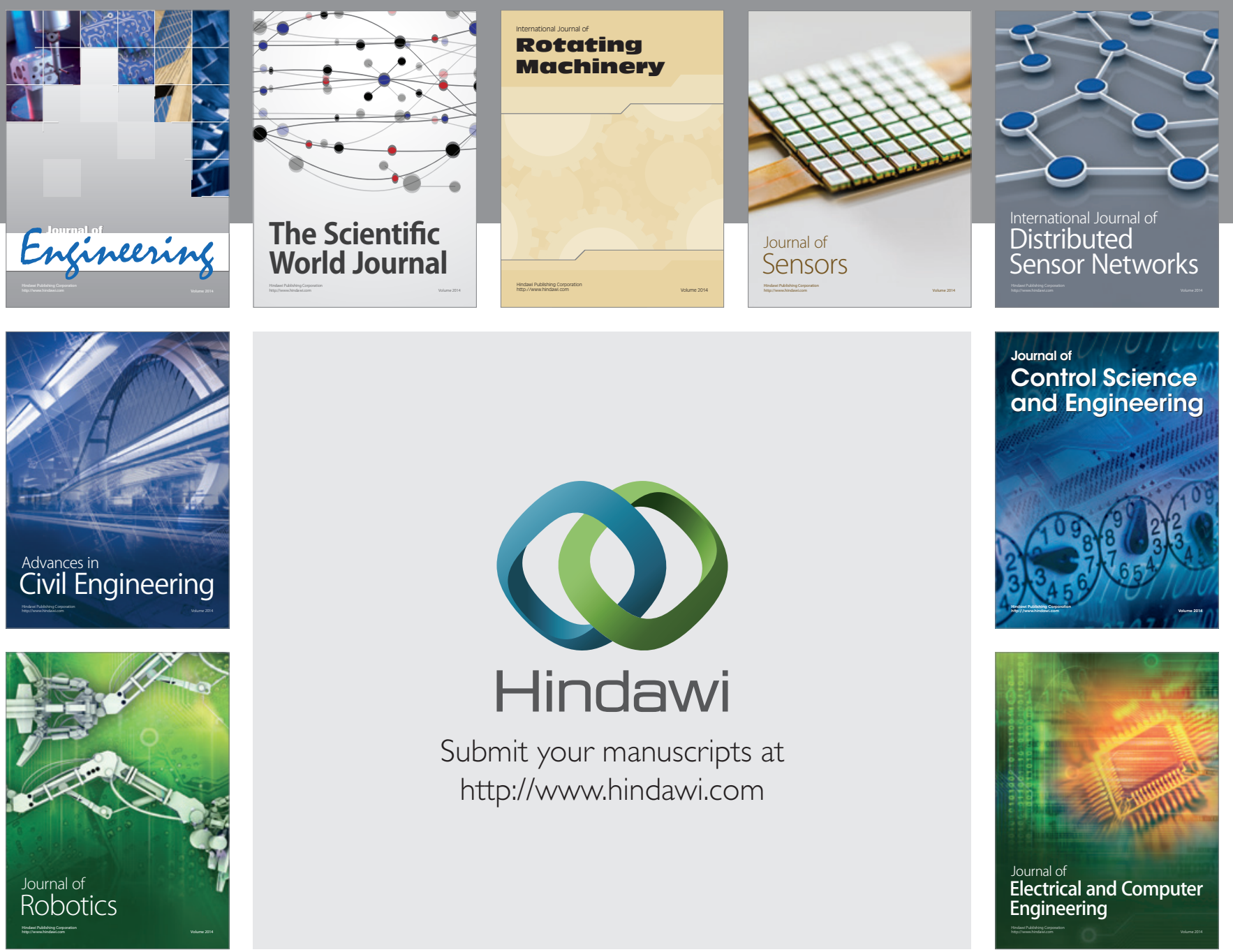

Submit your manuscripts at

http://www.hindawi.com
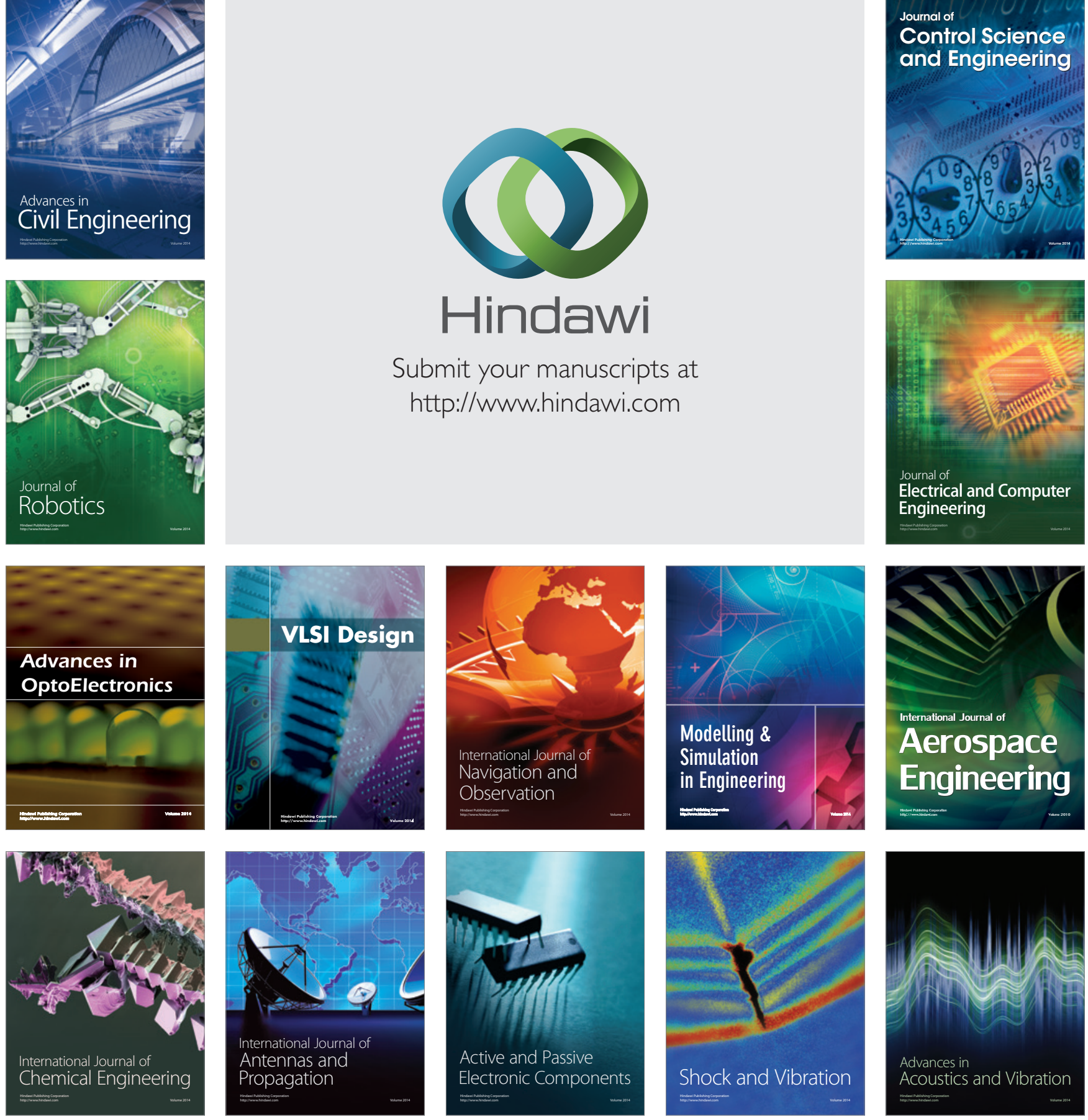